Präv Gesundheitsf 2021 · 16:177-178

https://doi.org/10.1007/s11553-021-00869-2

Angenommen: 28. Mai 2021

(c) Der/die Autor(en) 2021

\section{Gabriele Buruck}

Professur Gesundheitsförderung und Prävention, Fakultät Gesundheits- und Pflegewissenschaften, Westsächsische Hochschule Zwickau, Zwickau, Deutschland

\title{
Emotionen und Gesundheit - Förderung von Kompetenzen und Strategien
}

Emotionen legen $\mathrm{zu}$ einem großen Teil fest, wie wir mit unserem sozialen Umfeld interagieren. Sie motivieren uns zu bestimmten Verhaltensweisen und haben Auswirkungen auf unser Wohlbefinden und unsere Gesundheit. Vor allem die psychische Gesundheit spielt unter den aktuellen Anforderungen der Arbeit eine entscheidende Rolle. Themen wie Digitalisierung, Homeoffice und die Coronapandemie zeigen eindrücklich, dass die Förderung der psychischen Gesundheit als zentrale Zielgröße der betrieblichen Gesundheitsförderung eine entscheidende Rolle für Lebensqualität und Gesundheitsverhalten spielt [4].

Um Entscheidungen oder Verhaltensweisen zu erklären, werden in den Gesundheitswissenschaften häufig sozial-kognitive Theorien und Modelle herangezogen $[1,8,9]$. Diese betonen den Einfluss von Wissen, Risikowahrnehmung, Einstellungen, soziale Normen oder Selbstwirksamkeit. Mittlerweile liegen aus der Psychologie auch Erkenntnisse vor, welche zeigen, dass Emotionen und deren Regulation eine unerlässliche Voraussetzung für Entscheidungen und Verhalten darstellen $[6,7,11]$. Es ist unumstritten, dass dabei zwischen Person und Kontext vielfältige Wechselbeziehungen stattfinden [5].

So müssen Beschäftigte in Gesundheitsberufen (z. B. Pflege und Rettungsdienst) gleichzeitig die eigenen Emotionen und die Emotionen der vielfältigen Akteure ihres Berufsfelds regulieren. Diese Herausforderungen treffen dann auf Berufsfelder, welche durch defizitäre organisationale Arbeitsbedingungen (z.B. Fachkräftemangel und Fluktuation, fehlende Aufstiegs- und Weiterbildungsangebote sowie geringe Vergütungen) gekennzeichnet sind [10]. Das Gelingen der Balance zwischen Stress und subjektivem Wohlbefinden stellt unter diesen Bedingungen einen entscheidenden Aspekt für eine gute psychische Gesundheit dar [4].

Vor diesem Hintergrund möchte das Schwerpunktheft psychologische Perspektiven aufzeigen, welche den Fokus auf die Betrachtung emotionaler Regulation als einen wichtigen Erklärungsbeitrag für subjektives Wohlbefinden und psychische Gesundheit diskutieren.

„Detached concern“ als eine emotionsregulierende Bewältigungsstrategie wird in dem ersten Beitrag von Lampert, Hornung und Glaser als beeinflussender Faktor für psychisches Wohlbefinden unter Berücksichtigung von Selbstwirksamkeit und Sinnerfüllung bei der Arbeit betrachtet. So soll es Beschäftigten gelingen, in der Interaktion mit $\mathrm{Pa}$ tientengruppen und Angehörigen im Notfalleinsatz oder Arbeitsalltag eine Balance zwischen ausgeprägter empathischer Anteilnahme („concern“) und hinreichender Abgrenzungsfähigkeit („detachment“) zu finden.

In den weiteren Beiträgen des Schwerpunkthefts liegt der Fokus auf zwei relevanten Berufsgruppen der Gesundheitsberufe: Zum einen wird das Erleben von Stress und adaptiven (Problemlösen, Neubewerten, Akzeptanz) und maladaptiven (Unterdrücken, Grübeln, Vermeiden) emotionalen Strategien im Rettungsdienst von Behnke, Rojas und Gärtner beschrieben. Hier zeigt sich, dass im Rettungsdienst überwiegend adaptive emotionale Strategien angewandt werden. Insbesondere der Einsatz von Akzeptanz - im Sinne des Zulassens von Emotionen - geht mit weniger Belastungssymptomen einher.

Zum anderen zeigen Buruck und Haitsch, dass psychische Faktoren der Arbeit, emotionale Erschöpfung und emotionale Kompetenz einen relevanten Beitrag für psychisches Wohlbefinden im Rettungsdienst und in der Altenpflege leisten können. Gesundheitsberufe unterliegen seit Jahren einem zunehmenden Ökonomisierungsdruck. Die Regulation der eigenen Emotionen und der Emotionen des Gegenübers ist v.a. der Erfüllung des Arbeitsauftrags untergeordnet. Kann der Arbeitsauftrag nicht erfüllt werden, kann z.B. bei einem Rettungseinsatz der Patient oder die $\mathrm{Pa}$ tientin nicht gerettet werden, führt das zu vielfältigen Gefühlen wie Trauer, Angst oder Schuld. Die Auswirkungen auf das subjektive Wohlbefinden und damit die psychische Gesundheit der Beschäftigten hängen also auch entscheidend von der Flexibilität der gewählten Strategie ab, mit welcher alle auftretenden Emotionen bearbeitet werden [2].

Im Rahmen der Digitalisierung der Gesundheitsförderung gibt der Beitrag von Böhme und Berking einen Ausblick auf die Stärkung emotionaler Kompetenzen durch die Entwicklung einer Coaching-basierten Gesundheits-App. Basierend auf dem Training emotionaler Kompetenzen (TEK [3]) wird die Entwicklung einer gesundheitsbezogenen App vorgestellt, welche ergänzend oder unabhängig vom TEK zum Einsatz kommen kann. 
Innerhalb der Gesundheitsförderung spielen emotionspsychologische Betrachtungsweisen bisher eine eher untergeordnete Rolle. Unter Einbeziehung der aktuellen Entwicklungen in der Arbeit und der Kombination um Aspekte der gesundheitsförderlichen Arbeitsgestaltung stellen sie aber einen wichtigen Erklärungsbeitrag dar. Das Schwerpunktheft möchte hierzu durch die ausgewählten Artikel eine konstruktive Diskussion anregen. Ich danke allen Autorinnen und Autoren sowie den kritischen Gutachterinnen und Gutachtern, die mit ihren Arbeiten dazu beigetragen haben, dass die Diskussion zum Zusammenspiel von Emotionen und Gesundheit und die Förderung von Strategien und Kompetenzen der Emotionsregulation vorangetrieben wird.

\section{Korrespondenzadresse}

\section{Prof. Dr. Gabriele Buruck}

Professur Gesundheitsförderung und Prävention, Fakultät Gesundheits- und Pflegewissenschaften, Westsächsische Hochschule Zwickau

Am Kornmarkt, Zwickau, Deutschland

Gabriele.buruck@fh-zwickau.de

Funding. Open Access funding enabled and organized by Projekt DEAL.

Interessenkonflikt. G. Buruck gibt an, dass kein Interessenkonflikt besteht.

Open Access. Dieser Artikel wird unter der Creative Commons Namensnennung 4.0 International Lizenz veröffentlicht, welche die Nutzung, Vervielfältigung, Bearbeitung, Verbreitung und Wiedergabe in jeglichem Medium und Format erlaubt, sofern Sie den/die ursprünglichen Autor(en) und die Quelle ordnungsgemäß nennen, einen Link zur Creative Commons Lizenz beifügen und angeben, ob Änderungen vorgenommen wurden.

Die in diesem Artikel enthaltenen Bilder und sonstiges Drittmaterial unterliegen ebenfalls der genannten Creative Commons Lizenz, sofern sich aus der Abbildungslegende nichts anderes ergibt. Sofern das betreffende Material nicht unter der genannten Creative Commons Lizenz steht und die betreffende Handlung nicht nach gesetzlichen Vorschriften erlaubt ist, ist für die oben aufgeführten Weiterverwendungen des Materials die Einwilligung des jeweiligen Rechteinhabers einzuholen.

Weitere Details zur Lizenz entnehmen Sie bitte der Lizenzinformation auf http://creativecommons.org/ licenses/by/4.0/deed.de.

\section{Literatur}

1. Ajzen I (1991) The theory of planned behavior. Organ Behav Hum Decis Process 50:179-211

2. Aldao A, Sheppes G, Gross JJ (2015) Emotion regulation flexibility. Cogn Ther Res 39:263-278. https://doi.org/10.1007/s10608-014-9662-4

3. Berking M (2017) Training emotionaler Kompetenzen, 4. Aufl. Springer, Berlin

4. Badura B (2017) Arbeit und Gesundheit im 21. Jahrhundert. Mitarbeiterbindung durch Kulturentwicklung. Springer, Berlin

5. Grandey AA, Melloy RC (2017) The state of the heart: Emotional labour as emotion regulation reviewed and revised. J Occup Health Psychol 22:407-422. https://doi.org/10.1037/ocp0000067

6. Gross JJ (2015) Emotion regulation: current status and future prospects. Psychol Inq 26:1-26. https:// doi.org/10.1080/1047840X.2014.940781

7. Hochschild AR (1983) The managed heart: commercialization of human feeling. University of California Press, Berkeley

8. Rogers RW (1975) A protection motivation theory of fear appeals and attitude change. J Psychol 91:93-114

9. Schwarzer R, Lippke S, Luszczynska A (2011) Mechanisms of health behaviour change in persons with chronic illness or disability: the Health Action Process Approach (HAPA). Rehabil Psychol 56:161-170

10. BAuA (2020) Stressreport Deutschland 2019: Psychische Anforderungen, Ressourcen und Befinden. Bundesanstalt für Arbeitsschutz und Arbeitsmedizin, Dortmund

11. Zapf D (2002) Emotion work and psychological well-being: a review of the literature and some conceptual considerations. Hum Resour Manag Rev 12:237-268. https://doi.org/10.1016/S10534822(02)00048-7 